\title{
Diagnosis and outcome of subcortical cystic leucomalacia
}

\author{
J Q TROUNCE AND M I LEVENE \\ Neonatal Unit, Department of Child Health, Leicester University Medical School
}

SUMmARY Three children who had suffered prolonged hypotension or severe asphyxia and who subsequently developed cystic subcortical leucomalacia are reported. This condition fits into the spectrum of perinatal ischaemic brain disease but the diagnosis in life has not previously been reported. Aetiological factors and neurodevelopmental outcome, which was poor in all cases, are discussed.

The diagnosis of periventricular cysts using real time ultrasound is well documented. ${ }^{1}$ We now describe three infants with a very different distribution of cyst formation affecting the subcortical white matter, and discuss its importance.

\section{Methods}

Regular ultrasound examinations are performed on all infants of $1500 \mathrm{~g}$ birthweight and below as well as more mature infants with clinical neurological abnormalities. Scans were performed in coronal and parasagittal planes, through the anterior fontanelle using an ATL 300i real time scanner, with a 3.5, 5, and $7.5 \mathrm{MHz}$ multifrequency transducer. The lateral resolution of the $5 \mathrm{MHz}$ and $7.5 \mathrm{MHz}$ crystals are 1.6 and $0.9 \mathrm{~mm}$ respectively.

\section{Case reports}

Case 1. This girl was born by emergency caesarean section after placental abruption at term. Her birthweight was $2.9 \mathrm{~kg}$. She was severely asphyxiated and did not breathe spontaneously until 20 minutes after birth. Convulsions began on the first day of life and were initially well controlled by phenobarbitone. On day three there was an episode of extreme hypotension (systolic pressure $32 \mathrm{~mm} \mathrm{Hg}$ ) which responded to dopamine infusion. She subsequently developed further severe convulsions, profound hypotonia, and temperature instability. Cerebral ultrasound first showed subcortical cysts when the child was 6 weeks old (Fig. 1). Computed tomography, performed shortly after this, showed extensive areas of low attenuation in the cerebral parenchyma but no discrete cysts. Subsequent development was very abnormal with spastic quadriplegia, failure to suck, optic atrophy, and microcephaly. She died at the age of 3 months. Necropsy showed an atrophic brain particularly affecting the frontal lobes. There were multiple subcortical cysts (Fig. 2) corresponding in position to those seen on the ultrasound scan. In addition there was microcystic degeneration of both basal ganglia.

Case 2. This infant was born at 35 weeks' gestation by normal vaginal delivery; there had been meconium stained liquor and late decelerations on cardiotachograph for several hours before delivery. The infant weighed $2.46 \mathrm{~kg}$ at birth. Apgars 'ores were 3 at one minute and 7 at five minutes, and he did not need intubation at birth. He was subsequently ventilated for hyaline membrane disease, which was complicated by a pneumothorax. Sodium bicarbonate was used to correct an early metabolic acidosis. Convulsions occurred on the first day of life and were controlled by phenobarbitone and phenytoin. Postnatally the arterial blood pressure was always well maintained (systolic above $50 \mathrm{~mm} \mathrm{Hg}$ ). An ultrasound scan at the age of 12 days showed resolving intraventricular haemorrhage with mild ventricular dilatation. Two small periventricular cysts were seen together with generalised increase in echoes throughout the brain (Fig. 3(a)). Two weeks later extensive cystic degeneration was seen on ultrasound scan affecting both periventricular and subcortical regions (Fig. 3(b)). He was slow to start feeding and remained very irritable. At the time of 

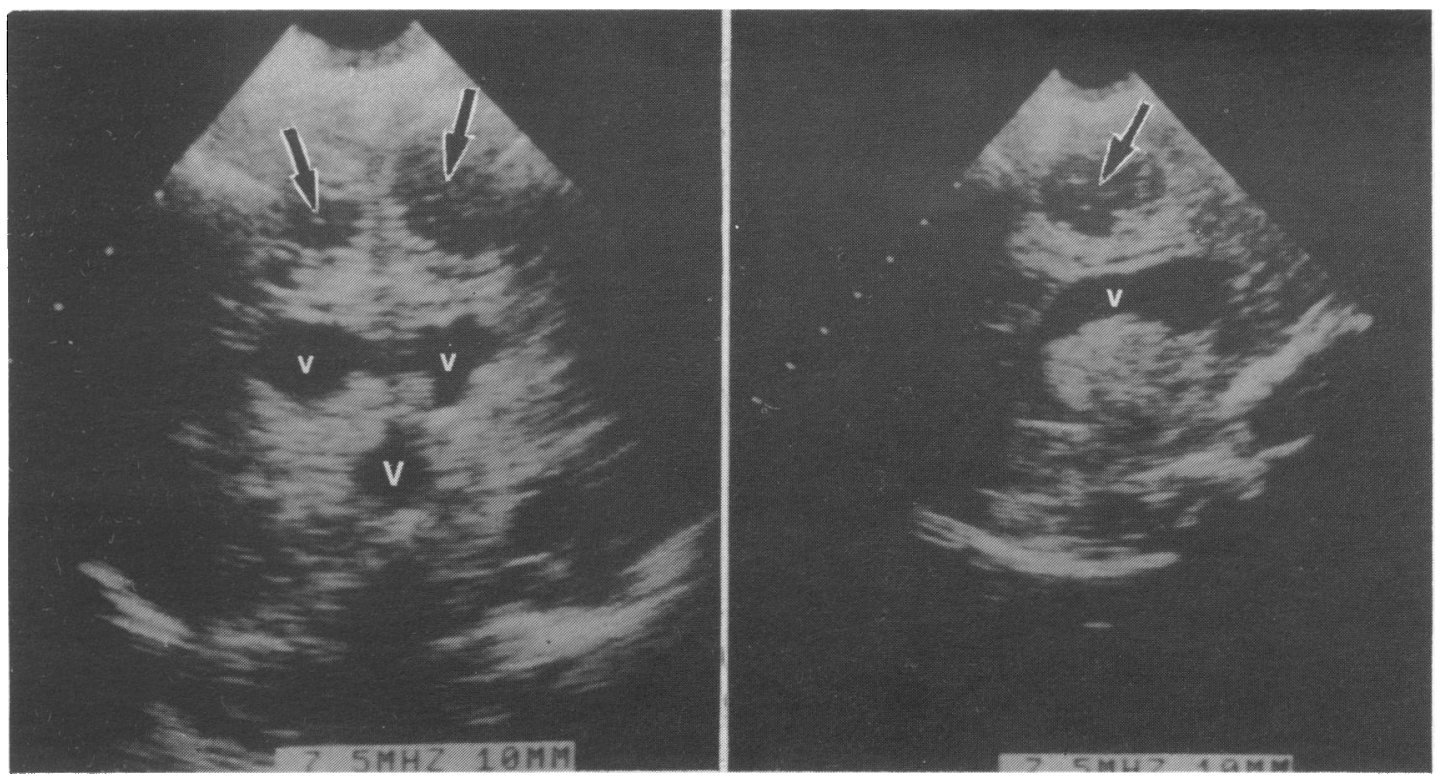

Fig. 1 Coronal ultrasound scan (left) and parasagittal scan (right) showing subcortical cysts in case 1.

There is irregular dilatation of lateral and third ventricles (marked with small $v$ and large $V$ respectively) consistent with cerebral atrophy.

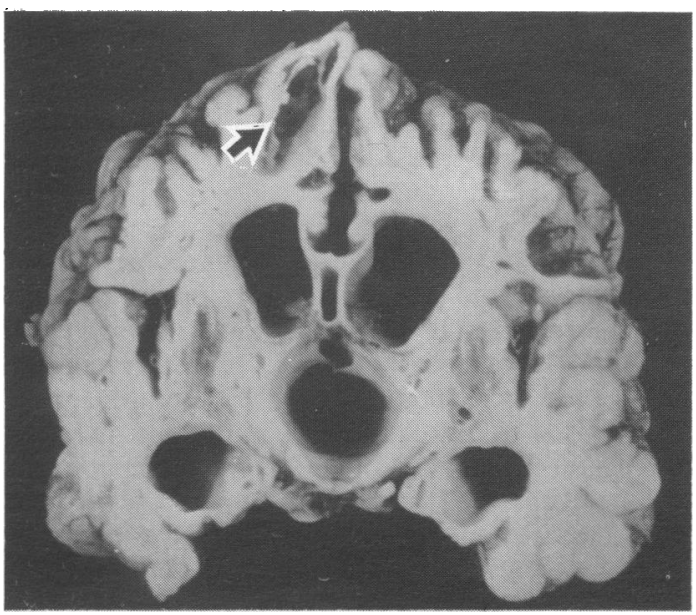

Fig. 2 Coronal section of the brain of case 1 .

There is a left sided subcortical cyst (arrowed), cerebral atrophy with enlargement of the lateral and third ventricles with basal ganglia degeneration.

writing he is 7 months old, suffers myoclonic seizures (controlled by nitrazepam), is still not smiling, and shows spastic quadriparesis with microcephaly.
Case 3. This patient was born by caesarean section after cord prolapse at 28 weeks' gestation. He weighed $1010 \mathrm{~g}$ at birth and was initially in good condition. He subsequently required ventilation for severe hyaline membrane disease, complicated by pneumothorax and patent ductus arteriosus. Persistent and profound hypotension (systolic blood pressure below $35 \mathrm{~mm} \mathrm{Hg}$ ) occurred between 18 and 31 hours of age, requiring plasma and dopamine treatment. The blood pressure was subsequently well maintained. Early ultrasound scans showed a small intraventricular haemorrhage and at the age of 12 weeks multiple subcortical cysts were first seen (Fig. 4). Computed tomogram showed symmetrical radiolucent areas confined to the parietal regions but no definite cysts were recognised. He has shown normal head growth and the occipitofrontal circumference currently lies on the 25 th centile (weight on the 3rd centile). His first social smile was at age 14 weeks. He is now 16 months old (corrected age 13 months), is hypotonic, and cannot sit unsupported. There is also impairment of fine motor control. $\mathrm{He}$ shows bilateral hearing loss on free field distraction testing, makes few babbling sounds, and uses no words with meaning. Although he had a convergent strabismus, his visual acuity is good. 

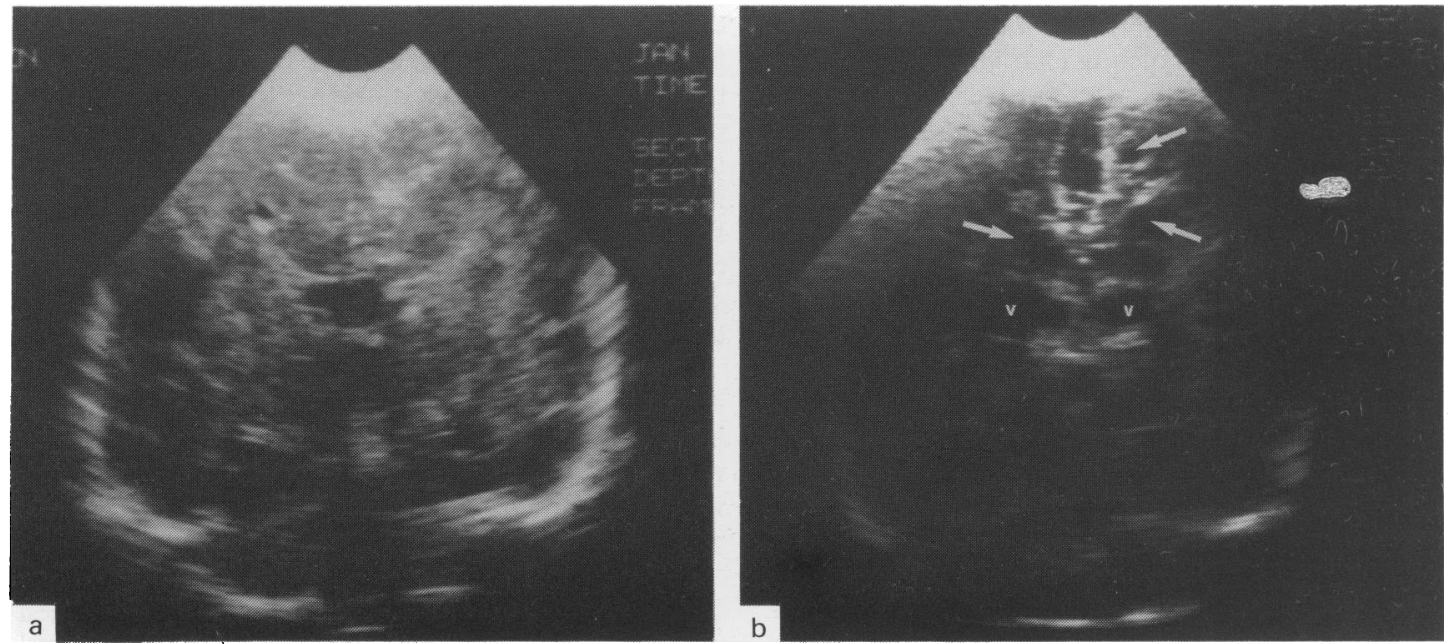

Fig. 3 (a) Coronal ultrasound scan of case 2 at age 12 days, showing a diffuse increase in echoes and a left sided periventricular cyst. (b) Two weeks later there is bilateral periventricular and subcortical cyst formation (arrowed). The interhemispheric fissure is widened and the lateral ventricles are minimally enlarged (marked with $v$ ).

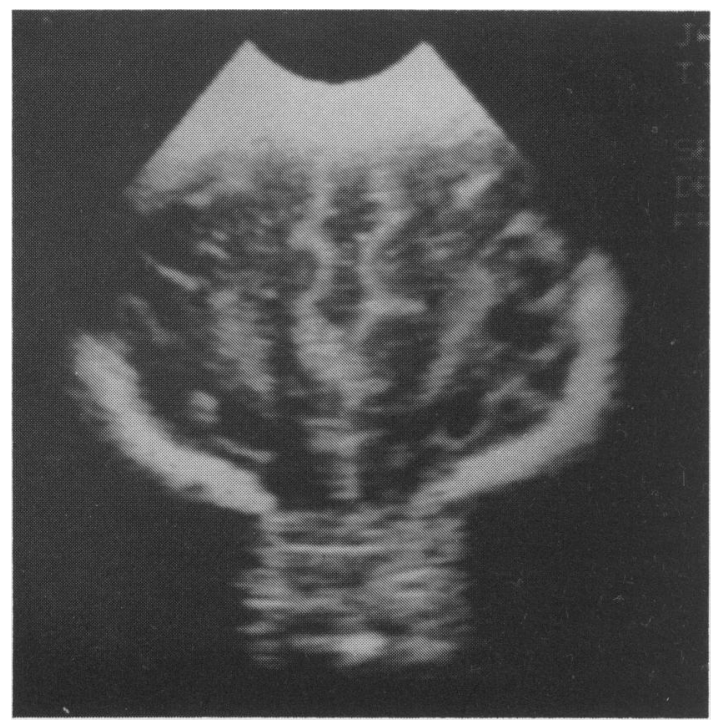

Fig. 4 Anterior coronal ultrasound scan of case 3 showing multiple subcortical cysts.

\section{Discussion}

Subcortical leucomalacia is one variant of neonatal hypoxic-ischaemic brain injury. The purpose of this report is to draw attention to the diagnosis by real time ultrasound, and the poor prognosis associated with it.

Subcortical leucomalacia occurs as a watershed injury at the boundary zones between the branches of the three major cerebral arteries. ${ }^{2}$ As term approaches there is regression of meningeal anastomoses between the major cerebral arteries which are normally present in the preterm infant. ${ }^{3}$ As a result of this, an important protective mechanism is lost and the subcortical region becomes a vulnerable site for ischaemic damage. In addition, the sulci deepen towards term and the penetrating vessels from the meningeal arteries bend acutely at the cortical-white matter junction as they pass towards the lateral ventricle. This leaves a relatively avascular triangle at the base of the sulcus which has been well shown by Takashima et $\mathrm{l}^{4}$ using post mortem microangiographic injection techniques. This region is particularly susceptible to damage as a sequel to decreased arterial perfusion. Brann and Myers ${ }^{5}$ have produced haemorrhagic necrosis of this area in newborn monkeys by experimental partial asphyxia. As a result of these two important changes in the cerebral circulation with increasing maturity, subcortical leucomalacia is much more likely to occur in term infants. The two mature infants reported here both suffered severe perinatal asphyxia, and one also had a period of severe postnatal hypotension. The third child reported, born at 28 weeks' gestation, suffered a period of profound and prolonged hypotension. We cannot explain why he showed this pattern of 
injury rather than the more common distribution of periventricular cysts which is characteristically seen in the preterm infant.

In addition to subcortical leucomalacia, infant 1 showed evidence of extensive cortical atrophy and basal ganglia degeneration. The second case also showed periventricular cysts. The poor prognosis in these children is therefore not surprising and may account for the different pattern of neurodevelopmental abnormalities seen in the third infant in whom no other abnormality was seen. The importance of subcortical cysts should not, however, be underestimated, as they provide a marker of severe brain insult, the aetiology of which seems to be intrapartum asphyxia or postnatal hypotension, or both.

Our experience with these three children suggests that subcortical cyst formation correlates with a poor neurodevelopmental outcome. At the time of her death, when aged 3 months, the first infant showed such severe cerebral damage that temperature control was impaired. The second child also shows serious sequaelae with myoclonic seizures, spastic quadriparesis, and microcephaly at the age of 7 months. Although the third child also shows delay in all aspects of development, his course has varied from that of the other two. At no time has he suffered convulsions and he now shows normal head growth and hypotonia rather than spasticity. This, perhaps, reflects the considerable difference in the response of the preterm brain to an insult when compared with its more mature counterpart.

Slovis reported a variety of parenchymal abnormalities seen on cranial ultrasound after perinatal hypoxic ischaemic insult. ${ }^{6} \mathrm{He}$ describes one infant of 32 weeks' gestation with multiple cystic encephalomalacia. This is a non-specific term for large cavitated lesions of the cerebrum which may result from various insults apart from asphyxia, such as meningoencephalitis or twin-twin transfusion. ${ }^{7}$ Babcock and Ball also reported this ultrasound abnormality in three of 18 asphyxiated term neonates. ${ }^{8}$ These reports, however, only emphasise the capacity of real time ultrasound to detect cystic changes within the cerebral parenchyma, rather than the aetiology of the cystic lesion. Ultrasound is now recognised as the best imaging technique for identifying periventricular cysts ${ }^{1}$ and it seems that other patterns of cyst formation may be equally well diagnosed. On computed tomography areas of hypodensity may be seen in the first two weeks after hypoxic-ischaemic insult and seem to be important prognostic pointers-90\% of asphyxiated term infants with this finding were abnormal at 18 months. ${ }^{9}$ Several workers have commented on the failure of computed tomography to show cystic changes that have been seen on a real-time ultrasound. ${ }^{810}$ In this study the presence of cystic lesions was not detected by computed tomography.

In conclusion, subcortical leucomalacia is an important variant of cerebral hypoxic-ischaemic injury, more commonly seen in the term infant, but which we also describe in one preterm neonate, and which is readily diagnosed by real time ultrasound. The prognosis seems to be very poor.

The authors thank Dr K Simpson, for permission to report clinical details of Case 2 and Dr Helen Grindulis, Senior Clinical Medical Officer, for performing detailed neurodevelopmental assessment on case 3.

Dr Trounce is supported by the Spastics Society.

\section{References}

${ }^{1}$ Levene MI, Williams JL, Fawer C-L. Ultrasound of the infant brain. Clinics in Developmental Medicine no. 92. London: SIMP, Blackwell Scientific Publications Ltd, 1985.

2 Smith JF. Pediatric neuropathology. New York: McGraw-Hill, 1974.

3 Volpe JJ. Neurology of the newborn. London: W B Saunders, 1981.

4 Takashima S, Armstrong DL, Becker LE. Subcortical leukomalacia. Arch Neurol 1978;35:740-2.

5 Brann AW, Myers RE. Central nervous system findings in the newborn monkey following severe in utero partial asphyxia. Neurology 1975;25:327-38.

6 Slovis TL, Shankaran S, Bedard MP, Poland RL. Intracranial hemorrhage in the hypoxic-ischemic infant: ultrasound demonstration of unusual complications. Radiology 1984;151:163-9.

7 Stannard MW, Jimenez JF. Sonographic recognition of multiple cystic encephalomalacia. AJNR 1983;4:1111-4.

8 Babcock DS, Ball W. Postasphyxial encephalopathy in full-term infants: ultrasound diagnosis. Radiology 1983;148:417-23.

9 Fitzhardinge PM, Flodmark O, Fitz CR, Ashby S. The prognositic value of computed tomography as an adjunct to assessment of the term infant with postasphyxial encephalopathy. J Pediatr 1981;99:777-81.

10 Martin DJ, Hill A, Fitz CR, Daneman A, Havill DA, Becker LE. Hypoxic/ischaemic cerebral injury in the neonatal brain. Pediatr Radiol 1983;13:307-12.

Correspondence to Dr M Levene, Neonatal Unit, Department of Child Health, Leicester Royal Infirmary, Leicester LE1 5WW.

Received 29 May 1985 